\title{
Las experiencias del personal docente de matemática en el trabajo de aula con la población no vidente
}

\section{The experiences of teachers of mathematics in classroom work with blind people}

\author{
Helen Bolaños-González \\ hellen.bolanos.gonzalez@una.cr \\ Escuela de Matemáticas \\ Universidad Nacional \\ Heredia, Costa Rica \\ Michael Céspedes-López \\ michael.cespedes.lopez@una.cr \\ Escuela de Matemáticas \\ Universidad Nacional \\ Heredia, Costa Rica \\ Cynthia González-Jiménez \\ cinthia.gonzalez.jimenez@una.cr \\ Escuela de Matemáticas \\ Universidad Nacional \\ Heredia, Costa Rica
}

Recibido-Received: 19/feb/2015 / Aceptado-Accepted: 4/abr/2015 / Publicado-Published: 31/ene /2016.

\begin{abstract}
Resumen
En este artículo se describe la perspectiva que tiene el personal docente de matemática en la educación superior sobre la atención integral de la población no vidente o de baja visión. Es de gran importancia recopilar las experiencias que ha tenido el profesorado de matemática de la Universidad Nacional con estudiantes que presentan alguna discapacidad visual y tener así un antecedente de lo que se ha hecho y los resultados obtenidos al aplicar ciertas estrategias metodológicas. Se busca representar la opinión del personal docente sobre los retos que debe enfrentar para llevar a cabo el abordaje metodológico y el proceso de evaluación de la enseñanza de la matemática. El desarrollo de la investigación se basa en el estudio de caso de un estudiante no vidente que ha reprobado el curso de cálculo diferencial e integral en varias ocasiones, por lo que se toma en consideración entrevistar al personal encargado de darle atención durante el II ciclo 2014. Basados en lo anterior, se analiza la información suministrada por la docente y el personal encargado de dar seguimiento y apoyo a los estudiantes con discapacidad visual. Tal información representa las experiencias vividas por el estudiante en los cursos del área de matemática y las principales características de su aprendizaje. Entre los resultados más relevantes de este trabajo se encuentran las características de la metodología y el proceso de evaluación empleados en una clase de matemática para estudiantes no videntes, así como las principales necesidades que presentan estudiantes con discapacidad visual en esta disciplina, con el objetivo de mejorar las condiciones favorables que se han venido dando para en la Escuela de Matemática de la Universidad Nacional de Costa Rica en relación con la educación inclusiva.
\end{abstract}

Palabras claves: Educación superior, matemática, estudiantes no videntes, estrategias didácticas. 
UNICIENCIA Vol. 30, No. 1, pp. 99-114. Enero-Junio, 2016.

ISSN Electrónico: 2215-3470

URL: www.revistas.una.ac.cr/uniciencia

DOI: http://dx.doi.org/10.15359/ru.30-1.6

Email: revistauniciencia@una.cr

\begin{abstract}
This article aims to present the point of view of professors of mathematics about the comprehensive care for the blind or low-vision population. It is important to collect the experiences that Mathematics teachers of the National University of Costa Rica have had with students with visual disabilities, and thus to have a history of what has been done, and the results obtained when applying some methodological strategies. This paper seeks to represent the teachers' opinion on the challenges they must face to carry out the methodological approach and the evaluation process of teaching Mathematics. The development of the research was based on a case study of a blind student who repeatedly failed the course of Differential and Integral Calculus, and therefore interviewing the staff responsible for giving him attention during the second semester 2014 was considered. Based on the above, the information provided by the teachers and the staff responsible for providing supervision and support to students with visual disabilities was analyzed. Such information represents the experiences of students in courses of Mathematics and the main characteristics of their learning. Among the most important results of this work are the features of the methodology and the process of evaluation used in a Mathematics class for blind students, as well as the major needs visually-impaired students have in this discipline, in order to improve the favorable conditions that the School of Mathematics of the National University of Costa Rica has been creating in relation with inclusive education.
\end{abstract}

Keywords: Higher Education, Mathematics, blind, teaching strategies .

El presente artículo nace como una experiencia de tres docentes de la Escuela de Matemática, quienes cursan estudios de posgrado en común y tienen como temática de su proyecto final las adecuaciones curriculares a estudiantes no videntes o con discapacidad visual en la educación superior en el área la matemática. Tema que ha sido escogido al identificar una necesidad que enfrentan el personal docente de la Escuela de Matemática de la Universidad Nacional (UNA), relacionada con la atención a esta población estudiantil.

A partir de los años setenta las universidades latinoamericanas, entre ellas la Universidad de Costa Rica (UCR) y, posteriormente, la UNA, comienzan a recibir a estudiantes con discapacidad (Moreno, 2005). El marco institucional y legal sobre la inclusión social de las personas con discapacidad de los países de América Latina muestra una tendencia regional a reconocer que la inclusión de personas con discapacidad en la educación superior es un asunto de derechos humanos.

Ante el aumento de la población con discapacidad visual que ingresa a la UNA y el derecho a la educación con el que cuentan, la Escuela de Matemática asume el reto, con mayor frecuencia durante los últimos años, de no solamente incluir en su población estudiantil a estas personas, sino también mejorar el proceso de enseñanza, por medio de la búsqueda de estrategias de trabajo conjunto con diferentes programas y proyectos y, así, ofrecer una educación de calidad de manera equitativa. Pues, tal y como menciona Moreno (2005), la inclusión de personas con discapacidad en la educación superior se focaliza fundamentalmente en el ingreso, pero no en la permanencia, progreso y egreso de los alumnos con discapacidad, aspectos en los que es necesario dar un paso como institución.

Es la Declaración de los Derechos Humanos y, las políticas y planes educativos de cada país, donde se establece que es responsabilidad del docente y parte del sistema educativo velar por el cumplimiento de este derecho y ofrecer, a la población estudiantil en general, una educación adecuada y de calidad.

Por esto, alrededor de los años 90, surge en América Latina la necesidad de incorporar temáticas relacionadas con la discapacidad y con ello se dan distintas iniciativas, como la 
ISSN Electrónico: 2215-3470

DOI: http://dx.doi.org/10.15359/ru.30-1.6
UNICIENCIA Vol. 30, No. 1, pp. 99-114. Enero-Junio, 2016. URL: www.revistas.una.ac.cr/uniciencia Email: revistauniciencia@una.cr

creación de la Red Interuniversitaria Latinoamericana y del Caribe sobre Discapacidad y Derechos Humanos, constituida en el año 2009. Esta tiene el objetivo principal de generar espacios educativos como entornos no excluyentes, propiciar el intercambio de experiencias, entre otros (Universidad de Buenos Aires, 2009).

A nivel nacional, Quijano (2008) afirma que se han realizado las acciones pertinentes para generar la igualdad de oportunidades estudiantiles, incluyendo estudiantes con necesidades educativas especiales asociadas a algún tipo de discapacidad, como lo es la visual.

El desarrollo de la educación especial en Costa Rica ha pasado por varias etapas, inicialmente, la creación de las escuelas de enseñanza especial y la inclusión de estudiantes que presentan algún tipo de discapacidad, principalmente en las aulas regulares de primaria y secundaria, abordadas por las llamadas adecuaciones curriculares. Mientras que en la educación superior, la inclusión de estudiantes con alguna discapacidad se encuentra aún en una etapa inicial y, respecto a ello, Berrios y Manosalva (2012) afirman que en el nivel de educación superior se cuenta con el menor porcentaje de personas con discapacidad estudiando, pero al mismo tiempo se evidencian mayores vacíos legales y de voluntades en lo que respecta a la integración de personas con alguna discapacidad.

De acuerdo con Correa (1999), una persona con alguna necesidad educativa debe ser considerada como alguien que presenta una forma particular de aprender, lo que no debe limitar sus posibilidades de desarrollo profesional para maximizar su potencial. Illanes y Von Furstenberg (2012) mencionan que el tema de las necesidades educativas especiales ha sido analizado con mayor frecuencia en los niveles de primaria y secundaria, no así en la educación superior; lo que conforma, en muchas ocasiones, una barrera tanto para estudiantes, como para docentes a cargo.

La formación de las personas con necesidades educativas tiene por objetivo principal la inserción social: el proceso no debería terminar en la enseñanza secundaria. La creciente población de estudiantes con dichas necesidades que finalizan la educación secundaria en Costa Rica, requiere opciones que le permitan, al igual que todos los otros grupos, una formación continua para enfrentar las demandas sociales y laborales. Sin embargo, en muchas ocasiones el desafío mayor lo tiene el personal docente en el aula, pues al mediar el proceso de enseñanza y aprendizaje en una formación profesional, la oferta académica debe ser de calidad para lograr una inserción social y laboral satisfactoria para tal estudiante con una necesidad educativa diferente, que requiere obtener habilidades y destrezas para su desenvolvimiento profesional.

Por lo anterior, en el presente trabajo se expondrán experiencias de docentes de matemática de educación superior, en el contexto de aula con estudiantes no videntes, con el propósito de analizar un estudio de caso, para lo cual es importante contar con referentes teóricos que, seguidamente, se expondrán.

\section{Referentes teóricos}

En este capítulo se tratará la concepción de adecuación curricular en educación superior, además se comenta la importancia de la formación universitaria de docentes de matemática y su incidencia para la atención de la población no vidente. Se considerarán las instancias de la universidad que ofrecen apoyo al personal docente y a estudiantes no videntes; así como la relación de estas y la coordinación de trabajo en conjunto dentro de la institución. 
UNICIENCIA Vol. 30, No. 1, pp. 99-114. Enero-Junio, 2016.

\section{Adecuaciones curriculares a nivel superior para la población no vidente}

El tema de las necesidades educativas especiales ha sido considerado de manera constante como objeto de estudio en la educación costarricense desde hace ya varios años, principalmente tratando el tema de las adecuaciones (significativas y no significativas) a nivel de primaria y secundaria. Sin embargo, se evidencia una falta de estudios que traten la inclusión de estudiantes con estas necesidades que terminan la educación media y desean acceder a un nivel de educación universitaria. Ello engloba uno de los retos que viven principalmente las universidades estatales, al promover enfoques de educación inclusiva para estos grupos de estudiantes y tratar de sensibilizar al personal académico sobre el derecho a la educación de estudiantes con discapacidad.

La presente investigación se enfocará en recopilar la experiencia que el personal docente de matemática ha tenido en el proceso de aula, al atender estudiantes que presentan necesidades educativas relacionadas con la ceguera o baja visión, de la UNA de Costa Rica. En la tabla 1 se muestra la cantidad de estudiantes con matrícula en las distintas carreras de la UNA, y que llevan seguimiento desde el proyecto "UNA Educación de la Calidad" adscrito en el Centro de Investigación y Docencia en Educación (CIDE).

Tabla 1

Estudiantes con discapacidad visual o baja visión de la Universidad Nacional, por carrera, 2014.

\begin{tabular}{llc}
\hline Escuela o Centro & Carrera & $\begin{array}{c}\text { Cantidad de } \\
\text { estudiantes }\end{array}$ \\
\hline Escuela de Matemática & Enseñanza de la Matemática & 2 \\
Escuela de Planificación y Promoción Social & Planificación Económica y Promoción Social & 4 \\
Escuela de Psicología & Psicología & 1 \\
Escuela de Literatura y Ciencias del Lenguaje & Enseñanza del Español / Francés & 3 \\
Escuela de Historia & Historia / Estudios Sociales & 2 \\
Escuela de Ciencias Biológicas Escuela de & Ingeniería en Bioprocesos Industriales & 1 \\
Química & & \\
CIDE: División de Educología & Arte y Comunicación Visual & 1 \\
Escuela Ecuménica y Ciencias de la Religión & Diplomado en Teología & 1 \\
Escuela de Administración & Administración & 2 \\
Escuela de Relaciones Internacionales & Relaciones Internacionales & 1 \\
CIDE: División de Educación Básica. & Pedagogía con Énfasis en I Y II & 1 \\
CIDE: División de Educación para el Trabajo & Orientación & 2 \\
CIDE: Maestría & Maestría en Educación Énfasis Universitaria & 1 \\
Escuela de Música & Educación Musical & 1 \\
Escuela de Economía & Economía & 1 \\
Total de estudiantes & - & 24 \\
\hline
\end{tabular}

Nota: (A. Fontana, coordinadora del Proyecto UNA Educación de Calidad. Comunicación personal, 18 de noviembre de 2014). 
De acuerdo con la información brindada por el proyecto UNA Educación de Calidad para el 2014, esta instancia atiende a 24 estudiantes con discapacidad visual matriculados en las distintas carreras. Este dato muestra el incremento de la población no vidente, que accede a la educación superior. En la tabla 1, se muestra que 8 estudiantes de nuevo ingreso, durante el año 2014, presentan alguna discapacidad visual y requieren apoyo de esta instancia.

Para el personal académico, esto debe ser un llamado de atención, pues deben estar preparados y capacitados para brindar una atención de calidad en los cursos de matemática, históricamente considerados cursos difíciles, lo cual no implica que no puedan ser accesibles a la población en general.

De acuerdo con Fontana, Espinoza y León (2009), existen varias definiciones de adecuación curricular, pero como señalan, todas coinciden en un punto y es la prestación de apoyos para que cada estudiante con necesidades educativas especiales pueda obtener éxitos académicos. En el 2005, el Centro Nacional de Recursos para la Inclusión Educativa (CENAREC) define las adecuaciones curriculares como una acomodación o ajuste de la oferta educativa a las características y necesidades de cada estudiante, con el fin de atender sus diferencias individuales. Por otra parte, establece que las adecuaciones de acceso son modificaciones o provisión de recursos especiales, materiales o de comunicación dirigidas a algunas poblaciones estudiantes (especialmente a aquellos con deficiencias motoras, visuales y auditivas) para facilitarles el acceso al currículo regular.

Asimismo, desde un enfoque de educación superior, Rodríguez, Andreu, Navas, Pereira, Rodríguez de Rivera, Sama y Sevillano (2010), respecto a la discapacidad visual la caracterizan por tener daños, en mayor o menor grado, ligados a factores susceptibles tales como la motricidad ocular, la visión cromática, la sensibilidad al contraste, la visión nocturna, entre otros. Además, cuantifican el grado de ceguera o de funcionamiento visual en dos variables: agudeza visual, habilidad para discriminar claramente detalles finos en objetos o símbolos a una distancia determinada; y campo visual, capacidad para percibir el espacio físico visible, cuando el ojo está mirando a un punto fijo.

En Costa Rica a lo largo del periodo comprendido desde el año 1973 hasta 1999 surgen varias leyes, las cuales marcan las pautas respecto a la educación de las personas con discapacidad, entre ellas: la Convención Americana sobre los Derechos Humanos (Ley 4534), la Ley sobre Igualdad de Oportunidades para Personas con Discapacidad en Costa Rica (Ley 7600), la Convención Interamericana para la eliminación de todas las formas de discriminación contra las personas con discapacidad (Ley 7948), la Convención sobre los Derechos Humanos de las Personas con Discapacidad (Ley 8661) y la Ley de Creación del Consejo Nacional de Rehabilitación y Educación Especial (Ley 5347). Estas brindaron la fundamentación jurídica que permitió la creación, en años posteriores, de la Política Nacional en Discapacidad (PONADIS).

La PONADIS aborda el derecho a la educación de las personas con discapacidad a partir del acceso universal y la equidad. Se considera la educación como el medio por el cual la población con discapacidad puede desarrollar y ampliar sus habilidades y destrezas, acceder a su desarrollo inclusivo y exigir el cumplimiento de sus derechos.

A través del proceso de admisión de la UNA, garantiza el ingreso a su programa en conformidad con las leyes y artículos establecidos a nivel nacional sobre igualdad de oportunidades para personas con discapacidad, por lo que, perfectamente, estudiantes con discapacidad visual pueden acceder a cualquier carrera que se oferte, siempre que aprueben el examen de admisión. Por otra parte, 
según la UNA (2014), en el Informe de Acreditación de la Escuela de Matemática en los últimos cuatro años, la carrera ha recibido a cuatro estudiantes con necesidades educativas especiales, específicamente con adecuaciones no significativas y dos estudiantes con discapacidad visual. Para el personal docente de la Escuela de Matemática se convierte en todo un reto atender a estudiantes con adecuación curricular; pero, más aún, si la necesidad educativa se basa en una discapacidad visual, pues durante la formación académica que recibe el profesorado de matemática, la preparación en el tema de necesidades educativas especiales es muy general.

\section{Instancias que ofrecen apoyo al personal docente o a estudiantes para la atención en adecuaciones curriculares}

La UNA, en el 2013, crea la Comisión Institucional en Materia de Discapacidad (CIMAD), la cual tiene como función promover principios de igualdad de oportunidades y accesibilidad para las personas con discapacidad, en todos los campus universitarios, reglamentos, políticas, planes, programas, proyectos, servicios, inversión, en el marco del plan de mediano plazo 2013-2017 y de la PONADIS.

La misión de la CIMAD está orientada a asesorar, planificar y articular los esfuerzos universitarios en materia de discapacidad; gestiona los cambios y ajustes institucionales para mejorar las condiciones de acceso y los servicios a toda la población; para ello, promueve cambios en las competencias en la comunidad universitaria, en la mejora de los servicios y facilidades (espacio físico, transporte, ayudas técnicas y apoyos tecnológicos); revisa y propone actualización de políticas, normas y procedimientos institucionales; gestiona estrategias de información y comunicación, en cuanto al desarrollo humano integral, incluyendo lo tecnológico y a lo jurídico. Asimismo, genera información histórica e indicadores institucionales sobre los temas asociados a su ámbito de acción.

La CIMAD tiene respaldo institucional en las Leyes 7600, 8661, 8862 dirigidas con los ejes temáticos del acceso e inclusión a la vida universitaria, innovación académica, sensibilidad y formación de la comunidad universitaria, acceso a la información y comunicación, entre otros.

A nivel institucional, la UNA (2013), en su Reglamento General sobre los Procesos de Enseñanza Aprendizaje de la UNA, en el Artículo 5, respecto a las Capacidades y Necesidades Educativas Especiales, dice que cada estudiante que presenta necesidades educativas derivadas o no de una discapacidad y que se encuentra inscrito en una carrera, tiene derecho a recibir lecciones accesibles de acuerdo con sus capacidades y necesidades e información sobre los servicios de apoyo que brinda la institución y la unidad académica.

Aunado a lo anterior, también existen los lineamientos institucionales para la digitalización de material para la persona con discapacidad visual, en el cual se acuerda emitir ciertos procedimientos para la digitalización, entrega y almacenamiento de documentos impresos, avalados por la CIMAD, que deberán seguir las facultades, centros y sedes para la utilización por parte de estudiantes con discapacidad visual.

Las leyes o reglamentos tanto institucionales como nacionales son claros en que se debe brindar el acceso a las personas con alguna discapacidad a la educación superior en igualdad de oportunidades. El cómo atender estudiantes no videntes en el contexto de clase en algunas oportunidades es difícil y es un reto para el personal docente que no cuenta con los conocimientos específicos del caso y herramientas óptimas para brindar una atención adecuada y de calidad a este tipo de estudiante, la formación que se le brinde debe concebir la necesidad de formarle integralmente para el trabajo de campo que fuese a desempeñar. 
Actualmente, parte del personal docente de matemática de esta universidad se ha enfrentado al reto de enseñar a personas con alguna discapacidad visual. En tales casos, reciben, adicionalmente, el apoyo por parte de personal tutor, asignado por el Programa de Éxito Académico de la UNA, mientras que el personal docente debe investigar sobre las metodologías, estrategias, el aprendizaje de lenguajes y demás para impartir su lección y no limitar el derecho a la educación de jóvenes con discapacidad. Aunque no es la totalidad de docentes que cuentan con estudiantes con problemas de visión en su salón de clases, cualquier profesor o profesora de la universidad podría, en cualquier momento, vivir esta situación.

El programa Éxito Académico pretende construir un proceso articulado y sistemático de apoyo y acompañamiento institucional que contribuya al desarrollo integral y egreso exitoso de estudiantes de la UNA. Esto, a través de tutorías para el fortalecimiento cognitivo que requieran. Se brinda la oportunidad de acompañamiento de tutorías planificadas por semana, ya sea de forma grupal o individual. Precisamente, entre los principales entes encargados de guiar al personal docente y al estudiantado con discapacidad en este proceso educativo en la UNA se encuentran el Departamento de Psicología y el proyecto UNA Educación de Calidad, los cuales se encargan de informar sobre lineamientos generales que debe cumplir el educador o educadora y los derechos estudiantiles. Ofrecen, al estudiantado, la oportunidad de recibir apoyo por parte de personal tutor, el cual es estudiante de niveles avanzados de la carrera de Enseñanza de la Matemática, además de un acompañamiento psicosocial, con el fin de darles seguimiento.

El Departamento de Orientación y Psicología se encarga de favorecer la integración y permanencia satisfactoria del estudiantado en la vida universitaria; la elaboración y desarrollo de decisiones de carrera adecuada, mediante los siguientes servicios: Primeramente, la consulta individual en orientación profesional. Este servicio consiste en un espacio para que cada estudiante comparta con una persona profesional sus inquietudes y situaciones, ya sea para la búsqueda conjunta de alternativas que contribuyan a su resolución, o bien para obtener asesoramiento en ámbitos como orientación vocacional, control del estrés, familia, sexualidad, relaciones interpersonales, entre otros. Otro de los apoyos recibidos es del Departamento de Orientación y Psicología que actúa como la unidad encargada de coordinar y organizar, a nivel institucional, las acciones y servicios que se ofrecen en la universidad para estudiantes con discapacidad. Esta atención brindada está muy de la mano del proyecto UNA Educación de Calidad. Tiene alrededor de 15 años de existir y su creación tuvo el propósito de superar las barreras actitudinales, físicas, sociales y culturales que limitan la participación plena de todos los grupos de estudiantes, prestando especial atención, al apoyo y seguimiento a la población estudiantil que presenta necesidades educativas en su formación universitaria. También, desarrollar un trabajo colaborativo dentro del proyecto para acompañar a estudiante y docente, además de desarrollar una estrategia y divulgar las acciones que permitan una inclusión social de esta población, lo que involucra un trabajo colaborativo entre los diferentes departamentos de la Vicerrectoría de Vida Estudiantil, con el fin de articular los apoyos que se proporcionen a la población estudiantil que lo requiera.

Paralelamente, el Programa Evaluación Académica y Desarrollo Profesional de la UNA integra la evaluación del personal académico y las acciones para el mejoramiento de docentes por medio de un Sistema de Desarrollo Profesional, programa que funciona desde el año 2007. El Sistema de Desarrollo Profesional se encarga de ofrecer, al personal académico, formación y 
actualización en diferentes áreas, con el fin de cumplir lo que dicta el Estatuto Orgánico, título III, del Régimen Académico, artículos 154 y 155.

En este apartado se ha pretendido brindar un claro panorama de los apoyos y servicios que brinda la Universidad Nacional y dejar en evidencia el compromiso que existe por mejorar esta vinculación y fortalecer la atención de la población no vidente, específicamente, en el área de la matemática.

\section{Descripción metodológica}

La investigación se enmarcó en el paradigma cualitativo, donde se aborda un estudio de caso cuyo propósito es describir y rescatar todas aquellas experiencias del personal docente así como la de quienes acompañan este proceso, en torno a la atención de un estudiante no vidente en un curso de matemática.

Por la naturaleza del estudio, se eligió el método de estudio de caso, desde una perspectiva fenomenológica, dada la temática a desarrollar dentro del contexto, ya que de esta manera permitirá tener una visión epistemológica y metodológica de lo que sucede con el estudiante.

La información analizada en la presente investigación se recolectó a través de entrevistas a profundidad aplicadas a personal docente de la Escuela de Matemática y personal del Proyecto UNA Educación de Calidad.

Las personas participantes de este estudio son, principalmente, docentes de la Escuela de Matemática de la UNA, que han tenido a cargo estudiantes no videntes. También se consideró el personal encargado del Proyecto UNA Educación de Calidad, el cual ha brindado información relevante para llevar a cabo el análisis detallado de las diferentes experiencias que se han generado particularmente en la Escuela de Matemática; pues tal proyecto, como acompañante tanto del personal docente como del estudiantado que así lo desee, tiene conocimiento sobre la mayoría de los casos y experiencias que atraviesan personal académico en conjunto con estudiantes que presentan discapacidad visual en la UNA.

Se consideró la entrevista abierta, ya que es un mecanismo que permite profundizar el conocimiento sobre una temática específica y su contexto. Para su desarrollo fue útil contar con una guía de conversación en la cual los tópicos son determinados de manera general. Nuevas preguntas y asuntos a tratar pueden ser resultado de la interacción con la persona entrevistada, lo cual es viable siempre y cuando no aparte del objetivo de indagación.

También se consideró la técnica de la entrevista en profundidad, puesto que la intención principal es adentrarse y detallar lo trascendente de las experiencias vividas por docentes de la Escuela de Matemática que deben atender a estudiantes con alguna discapacidad visual. Descifrar y comprender los miedos, las dudas, las satisfacciones, las angustias y las alegrías, significativas y relevantes de los participantes. Es decir, construir minuciosamente la experiencia del otro individuo, siguiendo un modelo de plática entre iguales, reuniones orientadas hacia la comprensión de las perspectivas de informantes respecto a sus experiencias o situaciones, tal como las expresan con sus propias palabras (Robles, 2011).

Por otro lado, se considera la técnica del análisis documental, que permite recopilar la información necesaria para elaborar un marco de referencia basado en las leyes y artículos que fundamentan y respaldan el derecho a la educación superior de las personas con alguna discapacidad visual y el deber del educador o educadora. Es decir, se materializan a través de un conjunto de procedimientos de análisis y síntesis, los documentos que tratan el contenido 
temático, con el objetivo de garantizar una recuperación precisa de los principales lineamientos que debe considerar el personal docente de la Escuela de Matemática para atender, en el desarrollo de un curso, estudiantes que presenten discapacidad visual.

\section{Análisis de resultados}

Se contemplaron a los cuatro docentes, que fueron profesores del curso de Cálculo I para este estudiante no vidente en particular. Para tal efecto se considerarán como Profesor 1, Profesor 2, y así consecuentemente; este ordenamiento responde al orden cronológico de los profesores en las diferentes oportunidades que el estudiante matriculó el curso antes mencionado. Además, es importante puntualizar que en una de estas oportunidades, el estudiante decide retirar el curso a inicios del ciclo, específicamente con el Profesor 2, por lo cual no se logran rescatar experiencias vividas por parte de dicho docente, en relación con la atención del estudiante. Aunado a ello, en las demás ocasiones, el estudiante logra finalizar el ciclo y aplicar los exámenes, pero no así cubrir la totalidad de contenidos ni aprobar el curso.

Para los profesores 1 y 3, se utilizaron entrevistas abiertas; para el Profesor 4 se realizó la entrevista a profundidad para conocer los detalles de su experiencia docente. Además, fue necesario realizar una entrevista a profundidad a la encargada del Proyecto UNA Educación de Calidad, debido a que esta entidad es parte fundamental del seguimiento de este alumno, dado que le brinda apoya en los cursos que corresponda.

Finalmente se consideraron algunos documentos y revisión bibliográfica de acuerdo con la necesidades vistas por los actores para mayor comprensión de la temática expuesta en este artículo.

\section{Principio de realidad del estudiante}

Lo descrito en este apartado es de acuerdo con la posición de los docentes y el personal del Proyecto UNA Educación de Calidad. Se detallan elementos claves para el análisis de caso que se presenta en este artículo.

El alumno padece deficiencia visual generada por desplazamiento de retina congénita. Este estudiante recibe apoyo del Proyecto UNA Educación de Calidad y del programa Éxito Académico con la asignación de un tutor, quien le dedica dos horas semanales al repaso de los contenidos. El alumno está inscrito en la carrera de Economía, lleva el primer curso de matemática en el año 2011, reprueba en dos oportunidades hasta que en la tercera vez logró aprobarlo. En el 2012 matrícula el curso de Cálculo I por primera vez; el Profesor 1 nos indica algunos obstáculos que afrontó el estudiante con respecto al acceso a la universidad, la inclusión y la socialización en el grupo, logra superar muchos de estos como deja ver con el Profesor 4.

La experiencia de educación secundaria fue muy marcada por el apoyo de sus padres, él proviene de una zona alejada, de una familia numerosa y de bajos recursos. En ese momento no aceptó recibir el apoyo de Instituto De Rehabilitación y Formación Helen Keller por decisión personal, lo cual no le permitió desarrollar destrezas en el manejo de recursos tecnológicos, lo que considera hoy una limitante, pues solo implementa el lenguaje braille para su proceso de enseñanza y aprendizaje, así lo señalan los entrevistados.

En primera instancia el Profesor 1 señala el traslado en las instalaciones universitarias complicado, pues si se daba un cambio de aula, el estudiante se perdía y no lograba ubicarse solo dentro del campus. En contraposición, dos años después se evidencia un cambio muy positivo 
al respecto. Al ingresar a la Universidad, se da una sobreprotección de sus padres, por lo que no lograba prepararse sus alimentos, entre otros obstáculos que logró superar, hoy en día es más independiente al movilizarse de su casa al campus universitario, así como su convivencia en las residencias es más autónoma.

Al entrevistar al Profesor 4, afirmó que aún persiste una dificultad en contenidos matemáticos y en el medio para comunicarse en la resolución de ejercicios; además menciona que el estudiante logra aprender fórmulas y métodos, pero no puede aplicarlos en ejercicios que demandan muchos pasos. De acuerdo con los profesores, se presentaba una dificultad mayor en el proceso aprendizaje, pues no lograba la comprensión de conceptos como, por ejemplo, el de límite, así lo señaló el Profesor 3.

Para apoyar el proceso de enseñanza y aprendizaje, el Programa Éxito Académico asignó para este II ciclo 2014 un tutor individualizado, quien comparte con él dos horas semanales en coordinación con el profesor a cargo, realizan un repaso de los contenidos que se vieron esa clase; sin embargo, el tiempo no es suficiente para el estudiante, así lo manifiesta el Profesor 4. El tutor digitaliza algunos materiales importantes para cubrir los contenidos del curso, lo cual es un gran apoyo para el docente y el estudiante.

De parte del Proyecto UNA Educación de Calidad se recomendó un cambio de carrera; sin embargo, se da un desacuerdo entre el estudiante, quien persiste en su elección de carrera.

Los docentes y el personal de apoyo consideran que es importante establecer relaciones favorables entre las instancias de apoyo a la labor docente para un proceso de enseñanza y aprendizaje con mayor claridad en la atención del alumno. Sin embargo, coinciden en que específicamente en el área de matemática existen vacíos por parte del estudiante que no corresponden al curso como tal, pero afectan considerablemente en la temática y ha sido muy difícil avanzar con el curso para el estudiante en relación con el resto de estudiantes matriculados.

\section{Retos del docente en matemática vrs adecuación curricular en educación superior}

En esta sección se describen los insumos obtenidos en las entrevistas realizadas a los docentes, para tal efecto se presentará el avance que el alumno ha tenido desde la primera vez que matriculó el curso con respecto a la más reciente.

El Profesor 1 nos describe algunos elementos claves de su experiencia de aula. Según menciona el Profesor 1, en ese momento no existía material alguno en braille para los temas del curso Cálculo I, lo que le complicaba la situación al estudiante. Poco a poco, se logra la transcripción de algunos materiales, por parte de las entidades competentes (en este caso asumió la responsabilidad el Proyecto UNA Educación de Calidad); sin embargo, el Profesor 1 enfatiza que los materiales eran tediosos, debido a que la parte teórica se trabajaba en un documento por aparte, separado de las gráficas y por un lado las fórmulas, en general se le tornaba complicado al estudiante unificar y relacionar todas las partes como un único tema. Además, el proceso de transcripción se inicia al comenzar el ciclo, por lo que el documento llegaba a las manos del estudiante de forma tardía, inclusive cuando el tema ya se había abarcado en totalidad; finalmente, al concluir el ciclo no dio tiempo de transcribir todos los materiales, por lo que el estudiante nunca contó con este recurso.

De acuerdo con lo expuesto por el Profesor 1, se torna difícil explicar los contenidos propios del curso Cálculo I, ya que el estudiante no presenta dominio de los contenidos del 
curso anterior, a saber, en temas de matemática básica como números reales, potenciación, operaciones algebraicas, entre otros, contenidos que se deben conocer desde secundaria; es decir, el estudiante no contaba con el manejo de conceptos elementales que se requerían para el nivel que se pretendía en el curso. Para lograr subsanar esta problemática, el docente decide extender el curso a dos ciclos, y así intentar cubrir con él estos vacíos. Sin embargo, el estudiante se limita a asistir solamente a las tutorías individualizadas, dejando de lado las clases presenciales, dado que no logra acomodarse al curso así como está planteado.

Por su parte, el Profesor 1 manifestó la necesidad de brindar mayor apoyo académico a este tipo de población, tarea que realiza el Programa Éxito Académico de la UNA. Sin embargo, sus instalaciones presentan un difícil acceso, tanto por su lejanía del campus Omar Dengo, como por su inadecuada infraestructura. Además, señala la dificultad que presenta al estudiante no vidente para ingresar a las instalaciones universitarias, pues, en este caso, el estudiante no conocía el camino para llegar a la Escuela de Matemática.

Respecto al planeamiento de la lección, y al haber extendido el curso de un ciclo a dos, el Profesor 1 afirmó que invirtió tiempo adicional a su jornada laboral, lo que le implicaba realizar dos planeamientos diferentes para cada lección; además buscar ayuda y asesoría, dentro y fuera de la universidad, pues considera no contar con capacitación específica para atender a la población no vidente en los cursos de matemática. Además, comentó que es necesario que exista un lugar o un departamento apropiado donde se pueda solicitar ayuda y el apoyo necesario para responder a esta necesidad en matemática.

Para el II ciclo del 2013, el estudiante matricula con el Profesor 2, dicho académico manifiesta estar en desacuerdo en una reunión con el Proyecto UNA Educación de Calidad, justifica no estar preparado para atenderlo de forma adecuada y solicita que se reubique en otro grupo, a consecuencia de esto el estudiante retira el curso y no lo vuelve a matricular sino hasta el I ciclo del 2014, lo que ocasiona que durante ese tipo no cuente con el acompañamiento por parte de la Escuela de Matemática ni del Programa Éxito Académico. Para el Profesor 3, el asunto de la falta de dominio de contenidos previos se vuelve una constante, hecho que al finalizar el ciclo no logra subsanarse. Aunado a ello, contaba con la limitación de no saber que tenía un estudiante no vidente en su lista de clase; por lo que no pudo brindarle la atención adecuada desde el primer día de clase. Además, el Profesor 3 manifestó necesitar capacitación en estrategias metodológicas para impartir el tema de análisis de gráficas a estudiantes no videntes, pues desconoce cómo enseñarle ese contenido a esta población.

Por cuarta vez, el estudiante decide matricularse en el curso, esta ocasión en el periodo del II ciclo 2014. Inicialmente el curso no estaba a cargo del Profesor 4, ya que estaba asignado a otro académico, lo que le permite en esta ocasión, al docente, conocer con anterioridad la existencia de un estudiante con discapacidad visual en su grupo. A partir de ese momento, se comunica con el estudiante, el cual le indica que se dirija al Proyecto UNA Educación de Calidad para mayor claridad de la situación específica del caso, por lo que se lleva a cabo una reunión donde se establecen algunos lineamientos del curso, muy generales; esto fue entre la primera y segunda semana lectivas.

El Profesor 4 afirmó llevar una bitácora por día, lo que da evidencia del trabajo realizado y la atención individualizada, en términos del planeamiento enfatiza que demanda mucho tiempo. Hace mención a que el braille es una herramienta que no logra cumplir su objetivo para expresiones algebraicas, pues el estudiante pierde muchos datos en el texto matemático en 
el momento de transcribir, en el caso específico de fracciones y potencias se evidencia que la herramienta entorpece el procedimiento, por lo que confunde al estudiante. Por ejemplo, en el tema de límites por racionalización, el estudiante pierde el hilo conductor de la resolución del ejercicio y deja datos olvidados. Otro ejemplo que señala es en el trabajo de integración por partes, donde el estudiante, a pesar de que entiende el procedimiento general, no logra resolver los ejercicios; se cuenta con una dificultada adicional: el documento teórico del contenido de integración no ha sido transcrito en braille, por lo que se le complica el acceso al estudiante; sin embargo, el tutor asignado en ese ciclo le digitó la tabla de fórmulas de integración.

Esta cuarta ocasión deja en evidencia que todavía existen vacíos en algunos temas; sin embargo, se refleja un mayor avance por parte del estudiante y apertura del docente para la atención. Al entrevistar al Profesor 4, este afirmó que siempre existió un diálogo fluido entre profesor y estudiante; no obstante, en las sesiones prácticas no se logró, debido a que el estudiante prefiere limitarse a grabar la clase, para posteriormente estudiar, ya sea de manera individual o con el apoyo del tutor asignado, situación que se ha evidenciado con los profesores anteriores.

Los docentes entrevistados, en su totalidad, manifiestan desconocer las estrategias de enseñanza que puedan emplear para atender a una persona con discapacidad visual en un grupo en promedio de 30 estudiantes que no tienen esa discapacidad. Señalan principalmente el desconocimiento de cómo enseñar matemática a una persona no vidente, y en segundo plano, dicha inclusión le genera al profesor atender a un mismo grupo de dos formas diferentes, pues las necesidades educativas del estudiante con discapacidad generalmente son muy distintas a las del resto de estudiantes.

Aunado a lo anterior, esto conduce a que el docente deba asumir la responsabilidad de sensibilizar primeramente al grupo, para que exista una mayor comprensión sobre las diferentes situaciones que se puedan presentar durante el desarrollo de la lección, como por ejemplo, una explicación más lenta y detallada por parte del profesor sobre los contenidos, con el objetivo que el estudiante con discapacidad visual pueda ir estructurando mentalmente un esquema de cada ejercicio.

La inversión de tiempo extra que debe hacer el docente en actividades adicionales, como lo son la atención individual adicional al tiempo de consulta que se ofrece al estudiante con discapacidad y al grupo en general, las horas adicionales en el planeamiento, las horas extras en la aplicación de prueba, entre otros, son aspectos que señalan los docentes que han tenido que asumir cuando tiene a cargo la atención de estudiantes con alguna discapacidad visual.

Entre los aspectos positivos que señalan los docentes se encuentran, primeramente, el acompañamiento de un tutor por parte del Programa Éxito Académico, encargado de atender dudas del estudiante con discapacidad visual y en varias ocasiones, importantes traducciones al braille del contenido y ejercicios de clase.

Según la información brindada por el Proyecto UNA Educación de Calidad y los docentes entrevistados, todos los estudiantes que presentan alguna discapacidad visual tienen diferentes maneras de aprender y diversas técnicas de estudio, lo cual genera en el profesor encargado la responsabilidad de identificar previamente una forma de enseñar congruente con el método de estudio del estudiante y el apoyo que recibirá de las distintas unidades de la universidad.

Relacionado con lo anterior, la construcción de estrategias metodológicas que se puedan implementar en la Enseñanza de la Matemática para estudiantes con discapacidad visual es considerada por los expertos de la UNA como un proceso muy variante. Pues ello dependerá del acceso con el que cuente cada estudiante a paquetes informáticos y sus actitudes para 
ISSN Electrónico: 2215-3470

DOI: http://dx.doi.org/10.15359/ru.30-1.6
UNICIENCIA Vol. 30, No. 1, pp. 99-114. Enero-Junio, 2016. URL: www.revistas.una.ac.cr/uniciencia Email: revistauniciencia@una.cr

aprender nuevas técnicas de aprendizaje, de estudio y demás. En los casos identificados, domina el desarrollo de una clase tradicional con un ritmo más lento, de forma tal que el estudiante con discapacidad visual puede realizar una construcción mental sobre el desarrollo del contenido, atención individual por parte del docente y de un tutor.

De acuerdo con el Proyecto UNA Educación de Calidad, las actitudes características del personal docente de matemática que han logrado generar un aprendizaje significativo en sus estudiantes con discapacidad visual es, primeramente, la actitud con la que se enfrenta la situación y la disposición tanto a colaborar en el proceso educativo, como recibir orientación en su quehacer educativo por parte de los diferentes programas con los que cuenta la UNA. En este proceso, es necesaria una actitud clara y positiva de docentes y estudiantes; en ocasiones de reiterada reprobación en un mismo curso, el Proyecto UNA Educación de Calidad ha realizado sugerencias como el cambio de carrera, principalmente, cuando se ha identificado la carencia de afinidad con la carrera que se cursa. También se valoran aspectos como la posibilidad de insertarse al mercado laboral.

El modelo de trabajo en el que se basa el Proyecto UNA Educación de Calidad para buscar un proceso educativo exitoso es horizontal, es decir, es un proceso en el que se involucra personal docente, estudiante con discapacidad visual, programa y Proyecto Éxito Académico. El objetivo de este método de trabajo se basa en la construcción conjunta de actividades que guíen la metodología de enseñanza, y las técnicas de estudio y acompañamiento. Es necesario considerar que la inclusión de estudiantes con discapacidad visual en la educación superior no solo está constituida por una relación entre docente y estudiante, sino que requiere de un trabajo colaborativo y coordinado con otros actores claves de la comunidad universitaria. Y para ello es fundamental considerar el estilo de trabajo y la cultura organizacional, además de la estructura y organización interna de la propia carrera.

El rol del personal docente de matemática es muy importante en el adecuado desarrollo de la inclusión y especialmente en la implementación de adecuaciones para estudiantes que presenten alguna discapacidad visual. Pero aun cuando él es uno de los protagonistas en la implementación adecuada de las adecuaciones, debe contar con el apoyo de un equipo de profesionales con especialidad en la temática, además de un tutor o tutora para cada estudiante.

\section{Proceso de enseñanza y aprendizaje, contexto de aula}

Al consultarle al estudiante sobre el trabajo de aula que los profesores llevaron a cabo con él, coinciden en que formó parte del grupo, él se sienta adelante y el docente verbaliza todo lo que se escribe en la pizarra. Al respecto, el Profesor 1 menciona que el estudiante inicia asistiendo regularmente y luego no logra comprender al mismo ritmo de los demás y decide no asistir más a lecciones presenciales sino individuales; aunado a lo anterior, el Profesor 3 indica que hubo una asistencia más regular, pero con vacíos de temas del curso, es decir, se perdía en las explicaciones y, a pesar de que se brindaba la hora adicional, no era suficiente para ponerlo al nivel del grupo. Para el Profesor 4, la situación persiste; sin embargo, se da un avance en cuanto a la socialización de grupo, pues los compañeros lo incluyen, aprenden a dejar el campo fijo en las primeras filas; aunque él no participa en el trabajo grupal, en la solución de ejercicios se brinda mayor apertura del grupo, lo que no ha sido evidente en los otros momentos.

Al consultarles con respecto a los exámenes aplicados en el curso, los profesores coinciden en se realizaron en un recinto aparte, donde se le dividieron de forma diferentes los contenidos; 
al respecto, el Profesor 4 comentó la experiencia en la aplicación de las pruebas escritas: el académico le dicta cada pregunta, la cual el estudiante procede a copiar en braille y revisa que se haya transcrito de forma correcta, para su revisión. Luego el estudiante lee su respuesta y el docente toma apuntes para poder asignarle el puntaje correspondiente; el académico indica que esta situación no es muy apropiada, pero considera que debido a la falta del manejo del lenguaje braille, esa fue la estrategia que más se adecuaba.

Para este caso se divide el curso de la siguiente manera: cada tema se evaluará en un examen, en total cuatro pruebas escritas, según cree de esta forma podrá conocer las deficiencias y debilidades por tema, finalmente se concluye, al finalizar el curso, que carece del dominio de todos los temas. Para fundamentar dicha conclusión, el docente relata la experiencia del último examen; se procede a dictar la primera pregunta, la cual corresponde al análisis de una gráfica, y lo deja trabajar antes de dictar la siguiente; sin embargo, el estudiante dura alrededor de cuatro horas por lo que el docente le propone continuar otro día la prueba, dado que el factor tiempo y cansancio jugaban en contra del estudiante. Además, el estudiante manifestó nunca haber graficado anteriormente, ya sea en algún curso a nivel universitario o en secundaria, por lo que le provocó dificultades en el tema de aplicaciones de la derivada en particular; se debe recalcar que los profesores anteriores no lograron abarcar este tema.

\section{Conclusiones y discusión}

En esta sección, se enuncian las principales conclusiones o recomendaciones, dando respuesta a la necesidad investigativa para analizar qué se ha hecho en nuestro quehacer académico. Cabe destacar que dichos resultados no pueden generalizarse a nivel nacional, puesto que obedecen a ciertas condiciones descritas en este artículo. Sin embargo, constituyen un punto de referencia para realizar mejoras al proceso de enseñanza y aprendizaje de la matemática a nivel superior en la atención de población no vidente.

El estudio de diversos factores implicados en la educación superior, específicamente en el área de Matemática al trabajar con población no vidente, conlleva al planteamiento de algunas conclusiones que permiten conocer las experiencias docentes en el trabajo de aula. Teniendo como sustento los resultados obtenidos a partir del análisis, se han podido establecer las siguientes conclusiones.

Es pertinente fortalecer el trabajo en equipo y obtener información de los proyectos y programas dispuestos en las universidades, institutos o centros de formación técnica; esto permitirá conocer las experiencias, analizar los retos que tiene la educación inclusiva, construir agendas conjuntas y reflexionar para avanzar hacia una educación superior inclusiva.

Es necesario desarrollar políticas, estrategias y procesos, para que sean incluyentes y equitativos. Se refleja la importancia de promover la cultura de respeto a la diversidad y el reconocimiento de cada estudiante como sujeto, donde toda la comunidad, interactúe bajo estas premisas con el fin de ayudar y apoyar el proceso educativo y de inclusión de la población con discapacidad en la universidad.

Considerando el desafío a nivel institucional que esto representa, se debe enfrentar en miras a mejorar la calidad de los procesos de enseñanza y aprendizaje de la matemática.

No se cuenta con infraestructura adecuada que facilite un mejor desplazamiento y accesibilidad, y promueva un ambiente adecuado a sus necesidades. Además, se debe reflexionar sobre la imperiosa necesidad de brindar capacitaciones al personal académico donde se aborden 
lineamientos, elaboración de materiales didácticos, adaptaciones metodológicas específicas para desarrollar los contenidos de los cursos de matemáticas.

Además, se refleja la necesidad de la creación de una instancia donde se le brinde un apoyo completo e integral al personal docente para enseñar matemática a estudiantes no videntes a nivel superior.

De manera general, falta sensibilización docente para atención integral que se debe brindar a la población de discapacidad visual, para enfatizar la armonía de trabajo en el aula.

Surgen algunas recomendaciones para la aplicación de las adecuaciones de acceso para estudiantes que presentan deficiencias de tipo visual. Estas se menciona a continuación:

Facilitar material fotocopiado de los resúmenes delos contenidos desarrollados durantela lección; ampliar la letra, si es necesario; utilizar la técnica "tutorías entre compañeros" como apoyo para la lectura de ítems, textos, técnicas y prácticas; nombrar una persona tutora (docente o estudiante) para que le lea el material escrito durante las pruebas; de ser posible grabar en audio las preguntas a evaluar, así las podrá reproducir tantas veces como lo necesite, para escuchar y entender los ítems; dar tiempo adicional en las pruebas, especialmente si las pruebas son escritas en braille, facilitarle software específicos de acceso de la información, como lectores de pantalla; para el trabajo de aula, se recomienda enumerar los pasos para su mayor comprensión de la temática.

Es importante considerar los elementos presentados en este artículo como base para el quehacer académico en el aula. La matemática siempre se ha caracterizado por sus bajos resultados en la pruebas a nivel nacional y a nivel superior esta constante no deja de ser evidente; por lo que al enfrentar el reto de la atención a la población con discapacidad visual, se suma otra variable adicional a dicha situación.

\section{Referencias}

Berrios, C. y Manosalva, S. (2012). Inclusión de estudiantes con discapacidad en la educación superior. Estudios y experiencias en educación. Redalyc, 11 (22), 13-34. Recuperado de http://www.redalyc. org/pdf/2431/243125410001.pdf

Centro Nacional de Recursos para la Inclusión Educativa. (2005). Compilación de disposiciones reglamentarias y lineamientos con relación a la atención de las necesidades educativas especiales de las y los estudiantes 1997-2005. Costa Rica: Editorama.

Correa, J. (1999). Integración escolar para la población con necesidades educativas especiales. Bogotá: Magisterio.

Illanes, L. y Von Furstenberg, M. (2012). Implementación de un programa de Inclusión a la Educación Superior de jóvenes con necesidades educativas especiales por discapacidad cognitiva en la Universidad Andrés Bello. Perspectiva Educacional, 51(2), 72-90. Disponible en http://www. perspectivaeducacional.cl/index.php/peducacional/article/vie wFile/111/44

Fontana, A., Espinoza, A. y León, A. (2009). Implementación de las adecuaciones de acceso al currículo en las instituciones educativas costarricenses. Centro Nacional de Recursos para la Educación Inclusiva, CENAREC. Costa Rica.

Quijano, G. (2008). La inclusión: Un reto para el sistema educativo costarricense. Revista de Educación. 32(1), 139-155. Recuperado de doi http://dx.doi.org/10.15517/revedu.v32i1.528

Moreno, M. T. (2005). Informe sobre la educación en América latina y el Caribe. Paper presentado en Seminario Regional sobre la Integración de las Personas con Discapacidad en la Educación Superior en América Latina y el Caribe. Caracas: IESALC. 
Robles, B. (2011). La entrevista en profundidad: Una técnica útil dentro del campo antropofísico Cuicuilco. Redalyc. org, 18(52), 39-49. Recuperado de http://www.redalyc.org/pdf/351/35124304004.pdf

Rodríguez, V., Andreu, A., Navas, N., Pereira, A., Rodríguez de Rivera, I., Sama V. y Sevillano, E. (2010). Atención a los estudiantes con discapacidad en la universidad. Orientaciones para el profesorado. Colección: Universidad Sin Barreras. Madrid. España: Editorial UNED.

Universidad de Buenos Aires. (2009). Informe final de la Red Interuniversitaria. Recuperado de: UBA http://ubadiscapacidad.wordpress.com/2009/06/17/informe-final-de-la-red-interuniversitaria/

Universidad Nacional. (2013). Reglamento General sobre los Procesos de Enseñanza Aprendizaje de la UNA. Heredia, Costa Rica.

Universidad Nacional. (2014). Informe de Acreditación de la Escuela de Matemática. UNA, Heredia, Costa Rica.

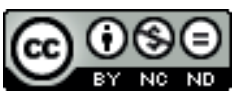

Las experiencias del docente de Matemática en el trabajo de aula con la población no vidente (Helen Bolaños-González y otros) por Revista Uniciencia se encuentra bajo una Licencia CreativeCommons Atribución-NoComercial-SinDerivadas 3.0 Unported. 